\title{
The severity of rat liver injury by fructose and high fat depends on the degree of respiratory dysfunction and oxidative stress induced in mitochondria
}

Claudia Isabel García-Berumen', Omar Ortiz-Avila, Manuel Alejandro Vargas-Vargas' ${ }^{1}$, Bricia A. del Rosario-Tamayo ${ }^{2}$, Clotilde Guajardo-López ${ }^{4}$, Alfredo Saavedra-Molina', Alain Raimundo Rodríguez-Orozco ${ }^{3}$ and Christian Cortés-Rojo ${ }^{1 *}$ (D)

\begin{abstract}
Background: High fat or fructose induces non-alcoholic fatty liver disease (NAFLD) accompanied of mitochondrial dysfunction and oxidative stress. Controversy remains about whether fructose or fat is more deleterious for NAFLD development. To get more insights about this issue and to determine if the severity of liver disease induced by fructose or fat is related to degree of mitochondrial dysfunction, we compared the effects of diets containing high fat $(\mathrm{HF})$, fructose $(\mathrm{Fr})$ or high fat plus fructose $(\mathrm{HF}+\mathrm{Fr})$ on NAFLD development, mitochondrial function, ROS production and lipid peroxidation.

Methods: Wistar rats were assigned to four groups: Control, fed with standard rodent chow; High fat (HF), supplemented with lard and hydrogenated vegetable oil; Fructose ( $F r)$, supplemented with $25 \%$ fructose in the drinking water; High fat plus fructose group ( $\mathrm{HF}+\mathrm{Fr}$ ), fed with both $\mathrm{HF}$ and Fr diets. Rats were sacrificed after 6 weeks of diets consumption and the liver was excised for histopathological analysis by hematoxylin and eosin staining and for mitochondria isolation. Mitochondrial function was evaluated by measuring both mitochondrial respiration and complex I activity. Lipid peroxidation and ROS production were evaluated in mitochondria by the thiobarbituric acid method and with the fluorescent ROS probe 2,4-H DCFDA, respectively.

Results: Fr group underwent the lower degree of both liver damage and mitochondrial dysfunction that manifested like less than 20\% of hepatocytes with microvesicular steatosis and partial decrease in state 3 respiration, respectively. HF group displayed an intermediate degree of damage as it showed $40 \%$ of hepatocytes with microvesicular steatosis and diminution of both state 3 respiration and complex I activity. HF + Fr group displayed more severe damage as showed microvesicular steatosis in $60 \%$ of hepatocytes and inflammation, while mitochondria exhibited fully inhibited state 3 respiration, impaired complex I activity and increased ROS generation. Exacerbation of mitochondrial lipid peroxidation was observed in both the Fr and HF + Fr groups.

Conclusion: Severity of liver injury induced by fructose or fat was related to the degree of dysfunction and oxidative damage in mitochondria. Attention should be paid on the serious effects observed in the HF + Fr group as the typical Western diet is rich in both fat and carbohydrates.
\end{abstract}

Keywords: NAFLD, liver steatosis, Mitochondria, Respiratory chain, Complex I, lipid peroxidation

\footnotetext{
* Correspondence: christiancortesrojo@gmail.com

${ }^{1}$ Instituto de Investigaciones Químico-Biológicas, Universidad Michoacana de

San Nicolás de Hidalgo, Edificio B-3, Ciudad Universitaria, 58030 Morelia,

Michoacán, Mexico

Full list of author information is available at the end of the article
}

(c) The Author(s). 2019 Open Access This article is distributed under the terms of the Creative Commons Attribution 4.0 International License (http://creativecommons.org/licenses/by/4.0/), which permits unrestricted use, distribution, and reproduction in any medium, provided you give appropriate credit to the original author(s) and the source, provide a link to the Creative Commons license, and indicate if changes were made. The Creative Commons Public Domain Dedication waiver (http://creativecommons.org/publicdomain/zero/1.0/) applies to the data made available in this article, unless otherwise stated. 


\section{Introduction}

Non-alcoholic fatty liver disease (NAFLD) is defined as excessive hepatic lipid accumulation in individuals whose alcohol intake is not significant and without any other hepatic disease. NAFLD encompasses a spectrum of liver alterations ranging from non-alcoholic fatty liver (NAFL), when steatosis is observed in absence of hepatocyte ballooning, non-alcoholic steatohepatitis (NASH), when steatosis is accompanied of ballooning and inflammation, to cirrhosis [1]. NAFLD is one of the most prevalent liver diseases in the world [2], affecting one third of the population in developed countries and increasing overall liver-related morbidity and mortality [3]. High-fat diets were initially believed to be the primary driver of the obesity epidemic [4], which was associated with increasing NAFLD prevalence [5]. Consequently, fat was substituted by fructose because this carbohydrate, in contrast with glucose, does not stimulate insulin secretion. However, fructose leads to liver stress due to excessive phosphorylation of this carbohydrate at the expense of ATP, causing phosphate deficiency, AMP accumulation, and increased synthesis of both uric acid and triglycerides $[6,7]$.

Diets with excessive amounts of fructose or fat induce hepatic mitochondrial dysfunction. Fructose increases the supply of electrons to the electron transport chain (ETC) by upregulating the tricarboxylic acid cycle [8], this along with impaired complex IV activity, increased mitochondrial levels of ROS and lipid peroxidation [9]. On the other hand, excessive fat intake impairs mitochondrial respiration in the phosphorylating state (i.e. mitochondrial state 3 respiration), inhibits complex IV activity, increases lipid peroxidation and augments ROS generation $[10,11]$.

The central role of mitochondrial dysfunction in NAFLD progression has been revealed by its alleviation with mitochondria-targeted strategies. For example, the deletion of the lysocardiolipin acyltransferase ALCAT-1 ameliorated NAFLD by inhibiting cardiolipin remodeling and improving both mitochondrial respiration and oxidative stress [11]. Furthermore, the alleviation of NAFLD in rodents by mitochondria-targeted antioxidants has been attributed to decreased lipid peroxidation, attenuation of oxidative stress and the inhibition of apoptosis [12].

On the other hand, it has been shown that diets containing high fat plus fructose induce more damage in both liver and mitochondria than a diet containing only high fat [13]. However, the effects of fructose alone were not compared with the effects of high fat or high fat plus fructose. This is an important issue to address, because the role of fructose in the development of metabolic syndrome has been put in doubt, thus there is not yet a definitive consensus about whether fat or fructose is more detrimental for NAFLD progression [14, 15]. Furthermore, it is unknown whether the severity of liver disease is related to the degree of mitochondrial dysfunction caused by fructose or fat. To address these issues, we have compared the effects of diets enriched in fructose (Fr), high fat (HF) and high fat plus fructose $(\mathrm{HF}+\mathrm{Fr})$ on NAFLD development, dyslipidemia, mitochondrial function, ROS levels and lipid peroxidation.

\section{Materials and methods}

\section{Animals and experimental groups}

Male Wistar rats weighing 250-350 g were used in this study. Each rat was housed in individual cages and maintained at room temperature with day/night cycles of $12 \mathrm{~h} / 12 \mathrm{~h}$, with free access to diets and water or fructose. Animals were randomly assigned to four groups (Table 1): 1) control group: fed only with standard rodent chow; 2) fructose group (Fr): fed with standard rodent chow plus 25\% fructose in the drinking water; 3 ) high-fat group (HF): fed with the HF diet; 4) high fat plus fructose group $(\mathrm{HF}+\mathrm{Fr})$ : fed with the HF diet plus $25 \%$ fructose in the drinking water. Diets were provided for 6 weeks. Food intake was limited to $20 \mathrm{~g}$ daily per rat to avoid the rancidity of not immediately ingesting food in the groups whose diets contained high fat. $250 \mathrm{~mL}$ of water or fructose solution was given daily to each rat, except that fructose was given to the $\mathrm{HF}+\mathrm{Fr}$ group every other day at the beginning of the 4th week, as its consumption decreased significantly at that time in this group. All the procedures with animals were performed according to the Federal Regulations for the Use and Care of Animals (NOM-062-ZOO-1999) issued by the Mexican Ministry of Agriculture.

High-fat diet (HF) was prepared with $47.5 \%$ standard rodent chow (Laboratory Rodent Diet 5001, LabDiet, St. Louis, MO, USA), 10.1\% lard, 40.6\% hydrogenated vegetable oil, $1.3 \%$ sodium cholate, $0.3 \%$ choline chloride and $0.2 \%$ thiouracil (Table 1 ). Standard rodent chow contained $23.9 \%$ protein $(28.5 \%$ of total calories), $5.0 \%$ fat

Table 1 Experimental groups and preparation of diets

\begin{tabular}{lllll}
\hline Diet components & \multicolumn{3}{l}{ Experimental groups } \\
\cline { 2 - 4 } & Control & $\mathrm{HF}$ & $\mathrm{Fr}$ & $\mathrm{HF}+\mathrm{Fr}$ \\
\hline Standard rodent chow ${ }^{\mathrm{b}}, \%$ & 100 & 47.5 & 100 & 47.5 \\
Lard $^{\mathrm{c}}, \%$ & & 10.1 & 10.1 \\
Hydrogenated vegetable oil $^{\mathrm{d}}, \%$ & 40.6 & 40.6 \\
Sodium cholate $^{\mathrm{e}}, \%$ & 1.3 & 1.3 \\
Choline chloride $^{\mathrm{e}}, \%$ & 0.3 & & 0.3 \\
Thiouracil $^{\mathrm{e}}, \%$ & 0.2 & & 0.2 \\
Fructose $^{\mathrm{f}}, \% \mathrm{w} / \mathrm{v}$ & & $25^{\mathrm{a}}$ & $25^{\mathrm{a}}$ \\
\hline
\end{tabular}

Diets were provided during 6 weeks to Wistar rats with an initial weight of 250-350 g. ${ }^{\text {a }}$ Fructose was given in the drinking water. ${ }^{\mathrm{b}}$ Laboratory Rodent Diet 5001, LabDiet, St. Louis, MO, USA. The composition of this diet can be consulted in [49]. 'JC Fortes, Empacadora San Benito, México. dManteca Inca,

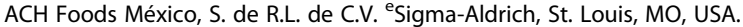

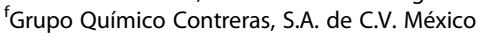


(13.4\% of total calories) and $48.7 \%$ carbohydrate $(58.1 \%$ of total calories). HF diet contained $14.7 \%$ protein $(8.8 \%$ of total calories), $53.8 \%$ fat (73.2\% of total calories) and $29.8 \%$ carbohydrate (18.0\% of total calories). Laboratory Rodent Diet 5001, which was used as the starting material to prepare the diets, exceeding several-fold the recommendations in vitamins and minerals made by the committee on AIN-93 purified diets for laboratory rodents [16], except for chromium, that is contained in similar concentrations in both diets, and for vitamin E, that is 1.78-fold higher in the AIN-93 diet than in the Laboratory Rodent Diet 5001.

\section{Isolation of mitochondria}

At the end of the treatments, rats were fasted $14 \mathrm{~h}$ and sacrificed by decapitation. The liver was excised and placed into ice-cold isolation solution (Medium 1) containing $220 \mathrm{mM}$ mannitol, $70 \mathrm{mM}$ sucrose, $1 \mathrm{mM}$ EGTA, and $2 \mathrm{mM}$ MOPS (pH 7.4). The liver was cut, washed, and homogenized with a Potter-Elvehjem homogenizer. The homogenate was centrifuged at 314 x g. Subsequently, the supernatant was decanted and centrifuged at $4410 \mathrm{x}$ g. The resulting pellet was washed with a solution (Medium 2) containing $220 \mathrm{mM}$ mannitol, $70 \mathrm{mM}$ sucrose, and $2 \mathrm{mM}$ MOPS ( $\mathrm{pH} 7.4$ ), and centrifuged at $6350 \mathrm{x}$ g. Finally, the pellet was re-suspended in $500 \mu \mathrm{l}$ of medium 2. Each centrifugation was performed during $10 \mathrm{~min}$ at $4{ }^{\circ} \mathrm{C}$ [17]. Mitochondrial protein concentration was measured by the Biuret method.

\section{Determination of biochemical parameters in serum and weight gain}

At the end of the six-weeks of treatments with diets, rats were fasted for $14 \mathrm{~h}$ and blood was recollected after the sacrifice for obtaining serum. Glucose, total cholesterol (TC), and triglyceride (TG) levels were measured by enzymatic methods with kits from VITROS Chemistry Products (Ortho Clinical Diagnostics Inc. Rochester, NY, USA), according to the manufacturer's instructions. Weight gain was determined by subtracting the weight of the animals after the six-weeks of treatments, measured right before sacrifice, minus the weight at the beginning of the treatments.

\section{Histological analyses of livers}

Small sections of the livers, obtained at the sacrifice of animals, were fixed in $10 \%$ formalin, embedded in paraffin blocks, sectioned ( $5 \mu \mathrm{m}$ thick) and stained with hematoxylin and eosin. Light microscopy was used for evaluation of steatosis, inflammation and hepatocyte ballooning. Microvesicular steatosis was evaluated like cytosolic accumulation of little lipid droplets not perturbing the central location of the nucleus. Macrovesicular steatosis was evaluated like cytosolic presence of large lipid drops that move the nucleus from its central position into the cell periphery [18].

\section{Evaluation of mitochondrial respiration}

Mitochondrial respiration was measured in basal, oligomycin-induced, state 4 (state $4_{\mathrm{O}}$ ) and phosphorylating state (state 3 ) by determining the oxygen consumption rate of freshly isolated mitochondria using a Clark-type electrode coupled to a YSI 5300A biological oxygen monitor and connected to a computer for data acquisition. $1.25 \mathrm{mg}$ of mitochondrial protein was placed into a sealed glass chamber containing respiratory buffer

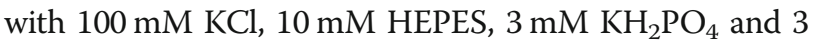
$\mathrm{mM} \mathrm{MgCl}_{2}$ (pH 7.4). The final volume was adjusted to $2.5 \mathrm{~mL}$. Respiration traces were started after adding mitochondria and $10 \mathrm{mM}$ glutamate/malate as respiratory substrate. State 3 was stimulated with $0.2 \mathrm{mM}$ ADP. State $4_{O}$ was induced by adding $1.4 \mu \mathrm{g} / \mathrm{mL}$ oligomycin. Respiratory control ratio (RCR) was calculated by dividing the respiration rate in state 3 vs the respiration rate in state $4_{\mathrm{O}}$.

\section{Determination of complex I activity}

$0.1 \mathrm{mg} / \mathrm{mL}$ of mitochondrial protein were resuspended in a buffer with $50 \mathrm{mM} \mathrm{KH}_{2} \mathrm{PO}_{4}(\mathrm{pH}$ 6.9) and incubated by $5 \mathrm{~min}$ with $1 \mu \mathrm{g}$ antimycin $\mathrm{A}$ and $1 \mathrm{mM} \mathrm{KCN}$ in a final volume of $1 \mathrm{~mL}$. Then, $5 \mathrm{mM} \mathrm{K}_{3} \mathrm{Fe}(\mathrm{CN})_{6}$ was added as electron acceptor and absorbance was registered at $340 \mathrm{~nm}$ in a Shimadzu UV2550 spectrophotometer. After $1 \mathrm{~min}$, NADH was added and its oxidation was monitored during $4 \mathrm{~min}$. The rate of $\mathrm{NADH}$ oxidation was calculated using the molar extinction coefficient of $16.3 \mathrm{mM}^{-1} \mathrm{~cm}^{-1}$ for $\mathrm{NADH}$ and the slopes of the time-traces of NADH oxidation.

\section{Measurement of ROS levels}

ROS levels were determined by measuring the oxidation of the fluorescent probe $2^{\prime}, 7^{\prime}$-dichlorodihydrofluorescein diacetate $\left(\mathrm{H}_{2} \mathrm{DCFDA}\right) .0 .5 \mathrm{mg} / \mathrm{mL}$ of intact mitochondria and $1.25 \mathrm{mM} \mathrm{H}_{2}$ DCFDA were incubated in a solution with 100 $\mathrm{mM} \mathrm{KCl}, 10 \mathrm{mM}$ HEPES, $3 \mathrm{mM} \mathrm{MgCl}$ and $3 \mathrm{mM} \mathrm{KH}_{2} \mathrm{PO}_{4}$ (pH 7.4) during $20 \mathrm{~min}$ at $4{ }^{\circ} \mathrm{C}$ under constant stirring. Then, mitochondrial suspension was placed into a quartz cuvette and basal fluorescence was recorded over time. After $1 \mathrm{~min}, 10 \mathrm{mM}$ glutamate/malate was added as substrate for the ETC and the changes in $\mathrm{H}_{2}$ DCFDA fluorescence were further monitored by $15 \mathrm{~min}$. Fluorescence changes were detected in a RF-5301PC spectrofluorophotometer (Shimadzu Corporation, Kyoto, Japan) $\left(\lambda_{\mathrm{ex}} 491 \mathrm{~nm}\right.$; $\lambda_{\text {em }} 518 \mathrm{~nm}$ ). ROS levels were calculated by subtracting the fluorescence $(\Delta \mathrm{F})$ detected after $15 \mathrm{~min}$ of substrate addition minus the fluorescence detected when substrate was added. $\Delta \mathrm{F}$ was divided then by the milligrams of mitochondrial protein used in the assay. 


\section{Lipid peroxidation assay}

Lipid peroxidation was evaluated with the thiobarbituric acid (TBA) method [19]. Mitochondrial pellets $(0.1 \mathrm{mg} / \mathrm{mL}$ protein) were washed twice and resuspended with $50 \mathrm{mM}$ $\mathrm{KH}_{2} \mathrm{PO}_{4}$ buffer ( $\mathrm{pH} 7.6$ ) immediately before TBA assay to avoid false positive results due to the interaction of thiobarbituric acid with the carbohydrates present in mitochondria isolation buffers. Lipid peroxidation levels were reported as thiobarbituric acid reactive substances (TBARS) per milligram of mitochondrial protein.

\section{Data analysis}

Data are expressed as mean \pm standard error of the mean. Statistical differences of data were determined with two-way analysis of variance (ANOVA), followed by multiple comparisons analysis performed with post hoc Tukey test. Statistical significance was set at $P<0.05$. Analysis were done with Sigma Plot 11.0 software (Systat Software, Inc., San Jose, CA, USA).

\section{Results}

Effects of fructose and fat on physiological parameters Control group showed a weight gain at the end of the study of $59.6 \mathrm{~g}$ (Fig. 1a). Higher weight gains of 82.7 and $80.7 \mathrm{~g}$ were observed in both Fr and HF groups, respectively. In contrast, there were no differences in weight gains between control and $\mathrm{HF}+\mathrm{Fr}$ groups. Regarding food consumption, there were no differences in this parameter among all the groups, since the animals fully consumed the amount $(20 \mathrm{~g})$ of food that was provided daily. No differences in water or fructose intake were detected within the groups, as all the rats drank the 250 $\mathrm{mL}$ that were given daily, except for the $\mathrm{HF}+\mathrm{Fr}$ group, which started to drink $\sim 110 \mathrm{~mL}$ fructose at the beginning of the 4th week of treatment.

Serum glucose concentration of the control group was $110 \mathrm{mg} / \mathrm{dL}$ (Fig. 1b) and lower levels were detected in the Fr, HF and HF + Fr groups, with values of 90.4, 91.6, and $68.5 \mathrm{mg} / \mathrm{dL}$, respectively. Serum triglycerides levels are shown in the Fig. 1c. In comparison to control group, triglycerides levels were 2.7- and 1.7-fold higher in both $\mathrm{Fr}$ and $\mathrm{HF}+\mathrm{Fr}$ groups, respectively, while no significant changes were observed in the HF group. Serum cholesterol levels (Fig. 1d) augmented moderately in the Fr group when compared to the control group. In contrast, cholesterol levels incremented up to 1.9 - and 1.7 - fold in the HF and HF + Fr groups, respectively.

\section{Effects of fructose and fat on liver histology}

Liver histological examination (Fig. 2 and Table 2) shows that livers from the Fr group displayed a lower percentage of hepatocytes with macrovesicular (10-20\%, black lines) and microvesicular (15\%, dotted arrows) steatosis, followed by the HF group with $40 \%$ of hepatocytes with
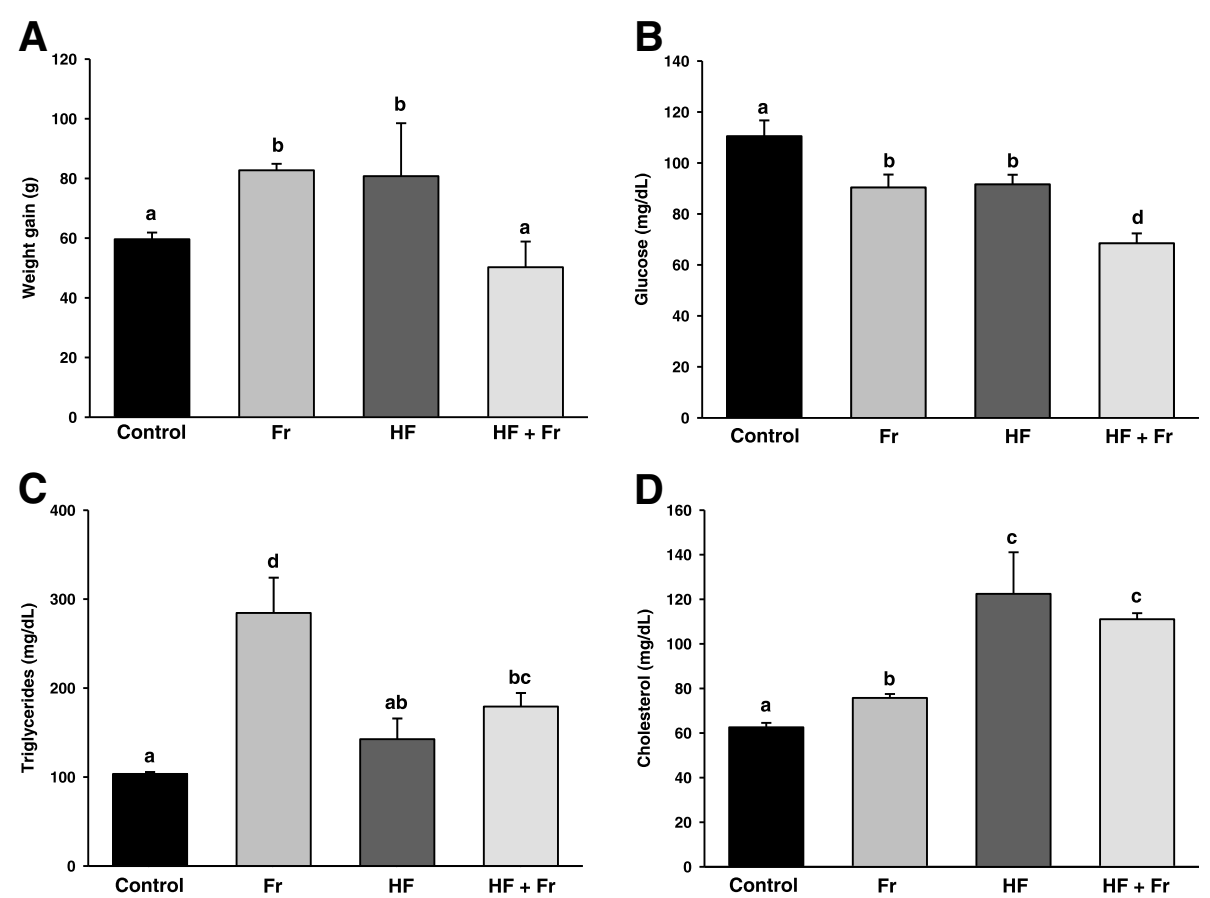

Fig. 1 Weight gain (a) fasting serum glucose (b), fasting serum triglycerides $(\mathbf{c})$ and fasting serum cholesterol (d) in rats that were fed for 6 weeks with diets containing normal rodent chow (Control), fructose (Fr), high fat (HF), and high fat plus fructose (HF + Fr). Weight gain was determined by subtracting the weight of the animals after six-weeks treatments with the diets, determined right before sacrifice, minus the weight at the beginning of the treatments. The results are presented as the mean \pm S.E. of $n \geq 4$. Different letters indicate statistically significant differences at $P<0.05$ 

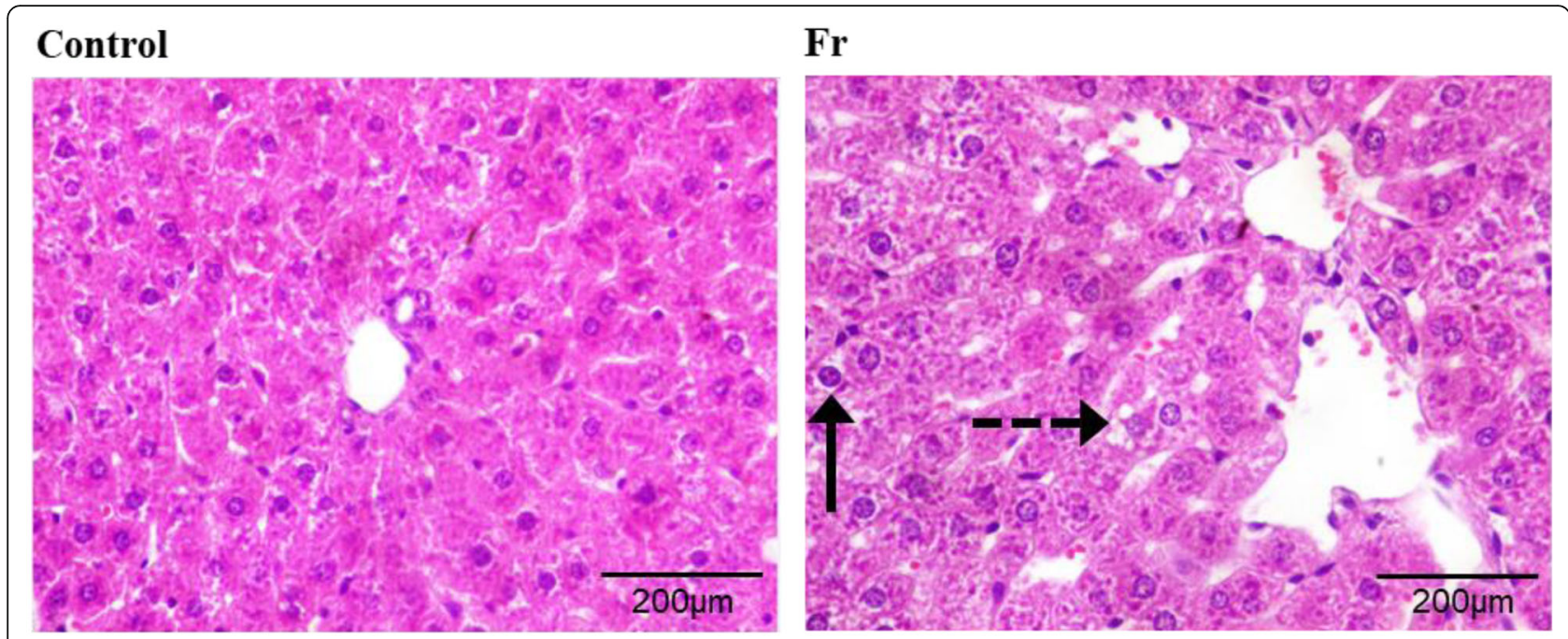

\section{HF}

\section{$\mathbf{H F}+\mathbf{F r}$}
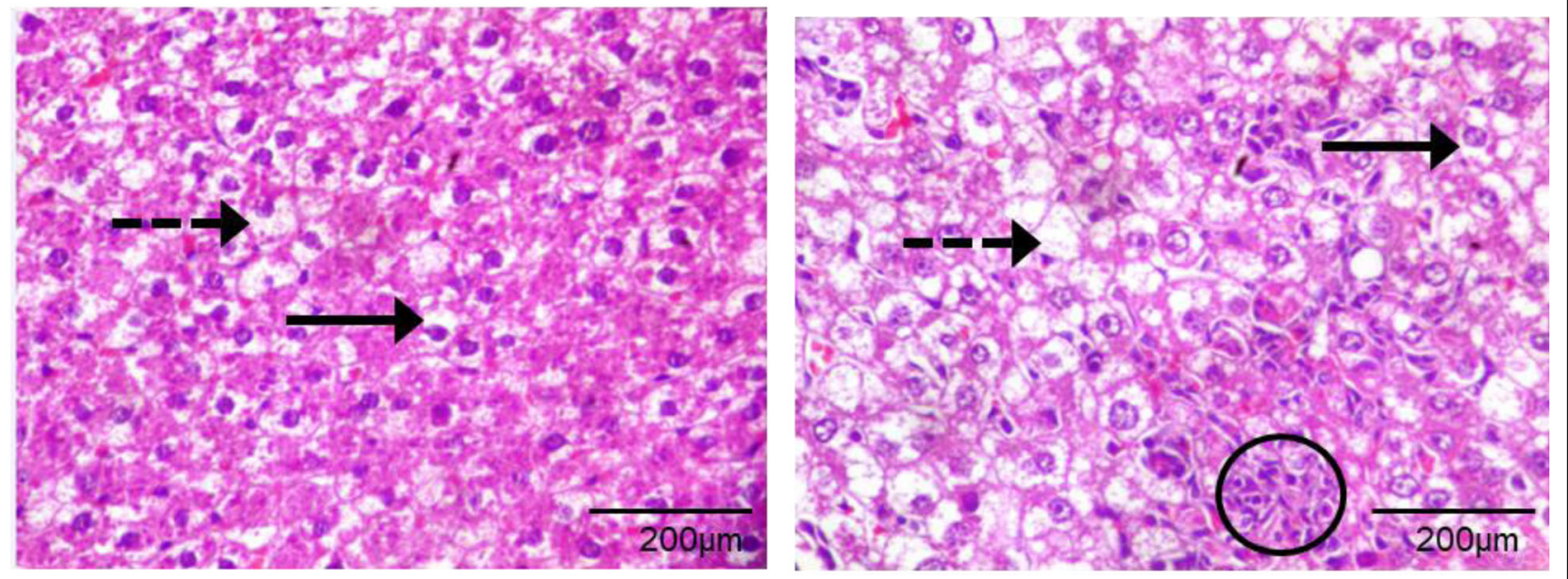

Fig. 2 Histological examination of livers from rats that were fed for 6 weeks with diets containing normal rodent chow (Control), fructose (Fr), high fat (HF) and high fat plus fructose (HF + Fr). Black arrows indicate the presence of macrovesicular steatosis; black dotted arrows indicate the presence of microvesicular steatosis; circle indicates inflammatory infiltrate. Liver sections were stained with hematoxylin-eosin and pictures were taken at 40X magnification

microvesicular steatosis and $60 \%$ with macrovesicular steatosis. The highest degree of steatosis was observed in the HF + Fr group, with $60 \%$ of hepatocytes with microvesicular steatosis and $80 \%$ with macrovesicular steatosis (in some cases, hepatocytes displayed both forms of

Table 2 Quantification of histological alterations induced in the livers of fructose (Fr), high fat (HF) and high fat plus fructose $(\mathrm{HF}+\mathrm{Fr})$ groups

\begin{tabular}{lllll}
\hline Liver alterations & \multicolumn{4}{l}{ Experimental groups } \\
\cline { 2 - 5 } & Control & $\mathrm{Fr}$ & $\mathrm{HF}$ & $\mathrm{HF}+\mathrm{Fr}$ \\
\hline Microvesicular steatosis & $1 \%$ & $10-20 \%$ & $40 \%$ & $60 \%$ \\
Macrovesicular steatosis & N.D. & $15 \%$ & $60 \%$ & $80 \%$ \\
Ballooning & N.D. & $60 \%$ & $60 \%$ & $70 \%$ \\
\hline
\end{tabular}

Data are expressed as the percentage of hepatocytes showing each type of alteration steatosis). Furthermore, the percentage of ballooned cells was $60 \%$ for both Fr and HF groups and $70 \%$ for the HF + Fr group. (Fig. 2). In addition, the HF + Fr group also showed chronic inflammatory infiltrate (circle).

\section{Effects of fructose and fat on mitochondrial function}

In mitochondria from the control group, the respiration rate was $\sim 7.7$-fold higher in state 3 than in state $4_{\mathrm{O}}$, thus resulting in a respiratory control ratio (RCR) of 7.7 (Fig. 3a). Respiration rate in state 3 decreased in the $\mathrm{Fr}$ and HF groups 1.6- and 1.4- fold, respectively, in comparison to the control group. This resulted in RCR values of 2.5 and 4.1, respectively. A 1.8-fold increase in state 4 respiration also contributed to the lower RCR observed in the Fr group. Rate of respiration in state 3 decreased 3.9-fold in the HF + Fr group when compared 

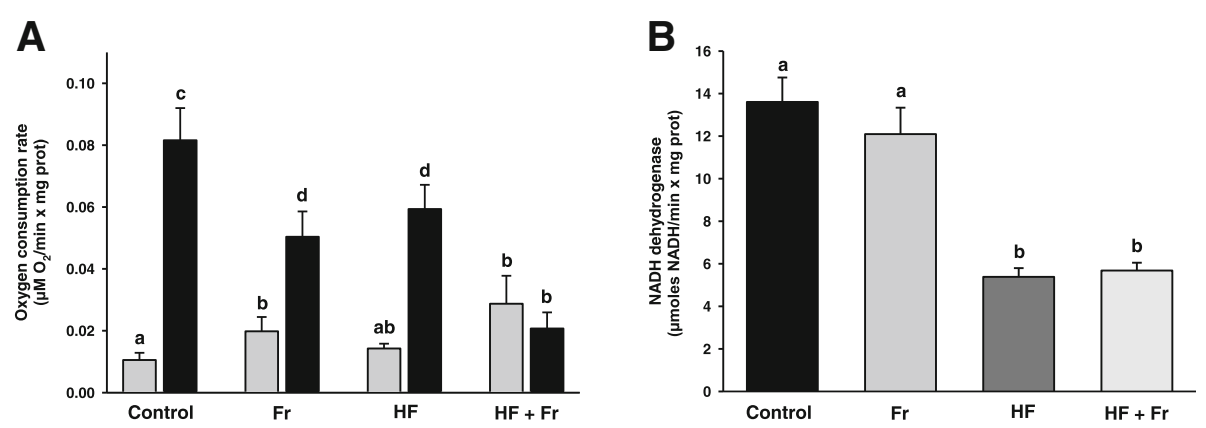

Fig. 3 Rate of respiration (a) and complex I activity (b) of liver mitochondria from rats that were fed for six weeks with diets containing normal rodent chow (Control), fructose $(\mathrm{Fr})$, high fat $(\mathrm{HF})$ and high fat plus fructose ( $\mathrm{HF}+\mathrm{Fr})$. Mitochondria were fueled with glutamate - malate. Respiration was measured in state 3 (black bars) and in oligomycin-induced state 4 (gray bars). The results are presented as the mean \pm S.E. of $n \geq 3$. Different letters indicate statistically significant differences at $P<0.05$

to the control group, while state 4 respiration increased 2.7-fold, which resulted in a RCR of 0.7 . On the other hand, complex I activity decreased $\sim 2.3$-fold in mitochondria for both the HF and the HF + Fr groups (Fig. 3b). In contrast, the activity in the Fr group remained unaltered.

\section{Effects of fructose and fat on mitochondrial ROS generation and lipid peroxidation}

Mitochondrial ROS levels doubled in the HF + Fr group in comparison to the control group (Fig. 4a). In contrast, no changes in the ROS levels were found in mitochondria for both the Fr and the HF groups. The levels of mitochondrial lipid peroxidation are presented in the Fig. 4b. In comparison to mitochondria of the control group, the levels of lipid peroxidation increased $\sim 8$ times in mitochondria from both the Fr and the HF + Fr groups, while no changes were detected in the HF group.

\section{Discussion}

Inclusion of fructose and/or fat in the diet induced a variable degree of hepatic damage. The $\mathrm{HF}+\mathrm{Fr}$ group exhibited the most negative outcome, as evidenced by the higher percentage of hepatocytes with microvesicular and macrovesicular steatosis and inflammation. The HF group showed an intermediate presence of steatosis; while the lowest percentage of hepatocytes with both types of steatosis was observed in the Fr group (Fig. 2 and Table 2).

As shown in Fig. 2, deleterious effects of fat were aggravated by fructose, since the HF group exhibited a lower extent of steatosis than the HF + Fr group (Fig. 2 and Table 2), besides the latter group also exhibited inflammation. In this regard, it has been hypothesized that the severity of liver damage correlates with the degree of oxidative stress in hepatocytes [20]. Accordingly, it was observed that the consumption of fat did not increase lipid peroxidation per se, as can be seen in the HF group (Fig. 4b). In contrast, fructose alone or combined with fat increased several-fold the levels of lipid peroxidation. Lipid peroxidation has been identified as an elicitor of NASH by triggering signaling cascades that mediates inflammation via an augmentation in the levels of malondialdehyde (MDA) and 4-hydroxynonenal (4-HNE), the end-products of lipid peroxidation [21, 22]. This suggest that lipid peroxidation induced by fructose might be triggering inflammation only when a higher degree of
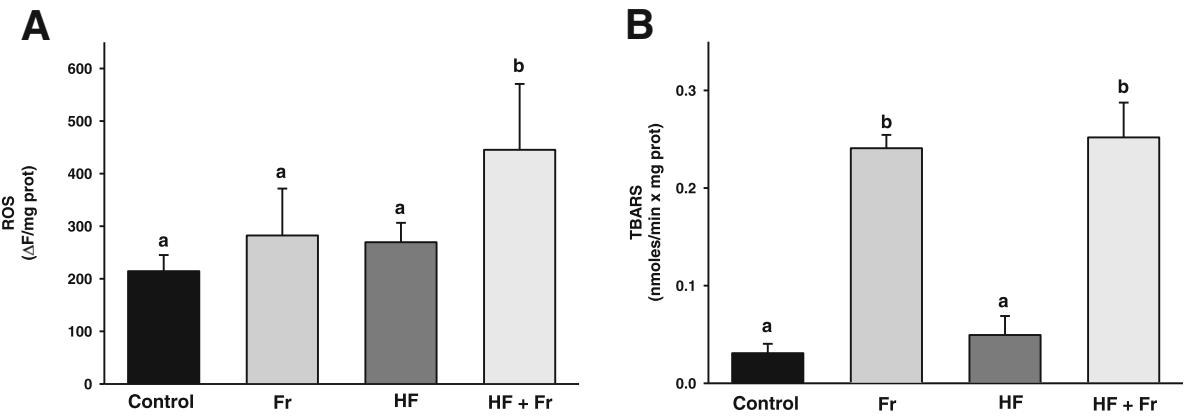

Fig. 4 Levels of ROS (a) and lipid peroxidation (b) of liver mitochondria from rats that were fed for six weeks with diets containing normal rodent chow (control), fructose (Fr), high fat (HF) and high fat plus fructose $(H F+F r)$. The results are presented as the mean $\pm S$.E. of $n \geq 3$. Different letters indicate statistically significant differences at $P<0.05$ 
steatosis is established by high fat intake. Nevertheless, it cannot be ruled out that an increase of lipid peroxidation and inflammation might occurs by the consumption of a HF diet over a longer period time. Despite the $\mathrm{Fr}$ group exhibited high levels of mitochondrial lipid peroxidation, it also showed a low level of steatosis without changes in ROS production, which suggest that mitochondrial lipid peroxidation per se does not cause severe liver damage in the absence of other factors such as significant accumulation of fat or increased mitochondrial ROS production as was only observed in the $\mathrm{HF}+\mathrm{Fr}$ group (Figs. 1 and 4a).

Another factor that may explain the presence of inflammation in the $\mathrm{HF}+\mathrm{Fr}$ group is the pronounced mitochondrial dysfunction resulting from a sharp decrease in state 3 respiration yielding a RCR of 0.7 (Fig. 3a), which can be interpreted as a full impairment of oxidative phosphorylation. ATP depletion due to both augmented fructose metabolism [7] and impaired oxidative phosphorylation (Fig. 3a) might lead to cell death by necrosis in livers of the $\mathrm{HF}+\mathrm{Fr}$ group, as ATP depletion is a hallmark of necrosis [23]. In turn, liver necrosis drives the release of intracellular content and activation of macrophages and neutrophils [24], which would be in concordance with the presence of inflammatory infiltrate seen in the HF + Fr group (Fig. 2).

A relationship was not found between the levels of lipid peroxidation and ROS, as lipid peroxidation increases in both the Fr and the HF + Fr groups, but ROS levels increases only in the latter one (Fig. 4). The absence of an apparent correlation among ROS and lipid peroxidation suggest that other factors different from ROS generation were responsible for increased lipid peroxidation by fructose. In this regard, induction of lipid peroxidation in liver by fructose has been associated to depletion of antioxidant defenses [25]. Lipid peroxidation is counteracted in mitochondria by phospholipid hydroperoxide glutathione peroxidase 4 (GPx4) using reduced glutathione (GSH) as an electron donor [26]. One catalytic round of GPx4 produces a molecule of oxidized glutathione (GSSG) that it is reduced back to two molecules of GSH by glutathione reductase (GR). It has been found that methylglyoxal (MGO), a reactive dicarbonyl involved in the production of advanced glycation end-products [27], inhibits the activities of GPx and GR [28]. Thus, a possible explanation for the several-fold increase of lipid peroxidation observed exclusively in the groups consuming fructose is that GPx4 and GR becomes inactivated by MGO produced during the hepatic metabolism of fructose.

Higher levels of ROS observed in mitochondria from the $\mathrm{HF}+\mathrm{Fr}$ group (Fig. 4a) correlates well with null stimulation of respiration by ADP that yielded an RCR of 0.7 (Fig. 3a). Decreased respiration due to inhibition of ATP production by $\mathrm{F}_{1} \mathrm{~F}_{0}$-ATP synthase leads to high rates of ROS production [29]. The lack of response to ADP addition can be interpreted like an impairment of $\mathrm{F}_{1} \mathrm{~F}_{0}$-ATP synthase to produce ATP, which leads to decreased electron transfer and increased ROS generation. Furthermore, the partial decline of state 3 respiration observed in both the HF and the Fr groups, and therefore, their RCR of 4.1 and 2.5, respectively, is in concordance with their lower levels of ROS with respect to the HF + Fr group. Overall, these data suggest that the main factor increasing ROS production was the full impairment of state 3 respiration, although it cannot be discarded that severe impairment in the activity of antioxidants systems (e.g. glutathione system) may also account for the higher ROS levels observed in the HF + Fr group.

Low rates of mitochondrial respiration in the liver increase the NADH/NAD ${ }^{+}$ratio [30], which leads to defective fatty acid $\beta$-oxidation and hepatic accumulation of triglycerides [31, 32]. This could explain exacerbated steatosis in the HF + Fr group (Fig. 2) as mitochondria from this group displayed the lower rate of state 3 respiration (Fig. 3a). Conversely, the lower severity of steatosis observed in the $\mathrm{HF}$ and Fr groups agree with their higher rates of state 3 respiration. On the other hand, complex I activity was lower in the HF group than in the Fr group (Fig. 3b); this might be involved in the higher degree of steatosis observed in the HF group by virtue of the role of complex I in NADH re-oxidation (i.e., the lower the complex I activity, the higher the NADH levels) and the dependence of fatty acid catabolism on low NADH/NAD ${ }^{+}$ratios. On this basis, it can be hypothesized that higher accumulation of fat in the livers of HF group was due to a low rate of NADH re-oxidation caused by a highly decreased complex I activity, while the $\mathrm{Fr}$ group had a low hepatic fat accumulation thanks to a fully functional complex I. In summary, there is an inverse relationship between complex I activity, the rate of state 3 respiration, and steatosis severity, which might be related to modulation of fatty acid $\beta$-oxidation rate exerted by $\mathrm{NADH} / \mathrm{NAD}^{+}$ratio and with the role of complex I activity on NADH redox turnover.

It has been considered that macrovesicular steatosis has a good prognosis when presented alone, with rare progression to NASH or cirrhosis. Microvesicular steatosis, by the contrary, is a less benign entity than macrovesicular steatosis because it has a serious prognosis and it is associated to impaired $\beta$-oxidation [33]. Taking into account the association between impaired $\beta$-oxidation and the presence of microvesicular steatosis, it can be postulated that excessive fat deposition in the $\mathrm{HF}+\mathrm{Fr}$ group might be largely due to the inability of mitochondria to re-oxidize NADH molecules produced during the $\beta$-oxidation, because low complex I activity in conjunction with fully inhibited oxidative phosphorylation. In 
the case of the HF group, intermediate fat deposition would be the result of low complex I activity along with partially functional oxidative phosphorylation, which may allow to re-oxidize NADH at intermediate levels. Finally, the relatively low deposition of fat in the $\mathrm{Fr}$ group would be attributed to higher capacity for NADH oxidation than in the other groups due to unaffected complex I activity and partially functional oxidative phosphorylation.

The pronounced deleterious effects of the HF + Fr diet are not trivial since the typical Western diet is abundant in high fat and high carbohydrates [34]. Besides, our results are consistent with another study showing that a high fat diet was significantly less deleterious than a high fat plus fructose diet, as the former induced only steatosis while the latter caused NASH. Furthermore, it was concluded that fructose was responsible for NASH development as the knockdown of the fructokinase gene prevented inflammation and fibrosis [35], which is also in line with our suggestion that fructose worsens the effects of fat by inducing necrotic cell death and inflammation. In contrast, it was shown in another report, that in comparison to our results, a high fat plus fructose diet had discrete effects on both respiration and lipid peroxidation in rat liver mitochondria [13], besides there were no differences between the effects produced by a high fat diet and the high fat plus fructose diet on these parameters. However, it must be stressed that in that work, diets were supplied for a shorter time (2 weeks) in comparison to this study (6 weeks), which may explain these different outcomes.

Several studies have shown that fructose does not affect weight gain [36-38], while others agree with our finding of increased weight by fructose [39-41]. Tillman et al. [38] have proposed that differences in weight response to fructose might be attributed to the way fructose was provided to animals (i.e. liquid or solid). For example, fructose was administered like aqueous solutions with high fructose corn syrup (HFCS) [39] or sucrose [41] in studies where fructose induced positive weight gain. In contrast, pelleted diet containing 60\% fructose was given in studies showing no alteration on body mass [36-38]. Nevertheless, in one of these studies where weight gain was observed, sucrose was given in solid form [40]. Thus, there is no clear relation between the physical form in which fructose is administered and its effects on weight gain. Another possibility might be the different effects that fructose-containing carbohydrates may have in anorexigenic hormones like leptin. Fructose promotes weight gain by decreasing leptin blood levels and dysregulating its actions in energy balance [42]. On the contrary, it would be expected that leptin actions on body weight were not so altered with the HFCS or sucrose-containing diets, since these carbohydrates also contains glucose, which may counteract the effects of fructose on leptin secretion by inducing insulin secretion. However, this explanation does not fit with the outcomes of the studies referred above, since sucrose or HFCS was given in the studies where increased weight gain occurred [39-41]. In contrast, fructose was administered in the studies where no differences in weight gain were observed [36-38], which conflicts with our finding about weigh gain with fructose (Fig. 1a). Thus, the possibility remains that Wistar rats, the strain used in this study, present a different phenotypical response to fructose ingestion with respect to body mass, which guarantee further research to compare the effects of HFCS, sucrose or fructose, given in aqueous solution or pelleted, on the body mass of rat strains with different genetic backgrounds.

It was unexpected that there were no differences in weight gain between the HF + Fr and the control groups, and that even weight gain of the $\mathrm{HF}+\mathrm{Fr}$ group was lower than that observed in both the HF and Fr groups (Fig. 1a). The most likely explanation for this finding is that fructose intake decreased more than $50 \%$ in the HF + Fr group at the beginning of the 4th week of treatment, which would be limiting calorie intake, and hence body weight gain. On the other hand, it can be hypothesized that lower weight gain in the HF + Fr group with respect to both the HF and the Fr groups may be due to combined result of exacerbated liver damage, highly impaired mitochondrial function, and a probable hyperinsulinemic status in rats of the HF + Fr group. Fructose is known to induce hyperinsulinemia [42]. Hyperinsulinemia in turn stimulates lipolysis in adipocytes and the release of free fatty acids into the bloodstream. Once in the liver, fatty acids are incorporated into triglycerides for their exportation in VLDL particles. Nevertheless, hyperinsulinemia also inhibits VLDL exportation to adipocytes [43], which may contribute in conjunction with increased lipolysis in adipocytes to decrease peripheric adiposity, as well to reduce fasting triglyceride levels as was observed in the HF $+\mathrm{Fr}$ group with respect to the Fr group (Fig. 1c). Probable hyperinsulinemic status might promote de novo triglyceride synthesis [44]; however, the deep impairment of oxidative phosphorylation in the HF + Fr group (Fig. 3a), and hence, the consequent failure in ATP synthesis, may counteract lipogenesis as this process is highly dependent on ATP [45], in this way leading to low availability of triglycerides to be exported to adipose tissue via VLDL. Probable inhibition of triglyceride synthesis also agrees with the severe degree of liver damage and higher levels of ROS observed in the HF + Fr group (Figs. 2 and 4a, respectively), as hepatic accumulation of free fatty acids due to inhibited lipogenesis produces oxidative stress and liver damage [44]. It may be argued against this hypothesis that the $\mathrm{Fr}$ group would have also exhibited a similar phenotype of 
decreased weight gain due to fructose-induced hyperinsulinemia. However, the different outcomes observed among the $\mathrm{HF}+\mathrm{Fr}$ and the Fr groups for the levels of glucose and triglycerides, and in weight gain suggest important differences between them in hormonal metabolic regulation. The idea that the $\mathrm{HF}+\mathrm{Fr}$ diet induces lower gain weight associated to hyperinsulinemia is supported by another study where reduction of weight gain, hyperinsulinemia and severe liver damage was found in rats fed with a $\mathrm{HF}+\mathrm{Fr}+$ ethanol diet [46], although this comparison must be taken with caution, as the degree at which ethanol contributes to this phenotype has not been elucidated.

Regarding the impact of diets on blood lipids, the more prominent effect was the increase in triglycerides in the $\mathrm{Fr}$ group (Fig. 1c), which agrees with the hyperlipidemic effect of this carbohydrate due to stimulation of de novo lipogenesis [47]. Unexpectedly, serum triglycerides in the $\mathrm{HF}+\mathrm{Fr}$ group were 1.6-fold lower than in the Fr group. A possible explanation for this observation is that the inhibition of oxidative phosphorylation in the $\mathrm{HF}+\mathrm{Fr}$ group (Fig. 3a) might be decreasing the rate of the de novo lipogenesis since the latter process is highly dependent on ATP produced by mitochondrial respiration [45]. On the other hand, the hypercholesterolemic effect of the diets containing HF (Fig. 1d) was not surprising due to the high content of cholesterol in the lard used for diets preparation.

A limitation of this study is that we did not determine if the severity of NAFLD induced by high fat or fructose is dependent on differential expression of pro-inflammatory cytokines like IL- 6 or TNF $\alpha$, which have been involved in NAFLD pathogenesis [48]. Likewise, we did not analyze the impact of the mitochondrial alterations observed with each diet on cytokine expression profile. Another limitation is that we did not examine the impact over NAFLD progression of inhibiting lipid peroxidation, ROS generation, or of counteract the impairment of oxidative phosphorylation. All that information would be useful to establish a causal link between the alterations induced by high fat or fructose in mitochondrial function and the progression of NAFLD. Another limitation is the lack of data about the $\mathrm{NADH} / \mathrm{NAD}^{+}$ratio, the hepatic triglyceride content, and the rate of mitochondrial $\beta$-oxidation, which would allow to verify the negative influence of fructose or fat in the mitochondrial utilization of fatty acids and its relationship with the severity of steatosis. Finally, we did not analyze the effects of fructose and high fat for longer time periods.

\section{Conclusions}

Despite producing the higher levels of lipid peroxidation, fructose provoked the less deleterious effects on both mitochondrial function and NAFLD since this carbohydrate partially decreased oxidative phosphorylation and induced the lower percentage of microvesicular steatosis. High fat exerted intermediate effects that manifested as decreasing in both oxidative phosphorylation and complex I activity in mitochondria, and intermediate levels of steatosis. The combination of high fat plus fructose produced the more deleterious effects in mitochondria including lipid peroxidation, enhanced ROS production, decreased complex I activity, and the full inhibition of oxidative phosphorylation, which fits well with the more severe liver damage induced by this diet that manifested as high levels of steatosis and inflammation. The latter might have implications for development of innovative strategies against NAFLD as the Western diet involves the simultaneous intake of excessive fructose and high fat. Hence, therapeutic approaches should be focusing in counteracting lipid peroxidation, excessive ROS production, and enhancing both oxidative phosphorylation and complex I activity.

\section{Abbreviations \\ 2,4-H 2 DCFDA: 2',7'-dichlorodihydrofluorescein diacetate; 4-HNE: 4- hydroxynonenal; ALCAT-1: acyl-CoA:lysocardiolipin acyltransferase 1; ANOVA: analysis of variance; EGTA: ethylene glycol-bis(2-aminoethylether)- $\mathrm{N}, \mathrm{N}, \mathrm{N}^{\prime}, \mathrm{N}^{\prime}$-tetraacetic acid; ETC: electron transport chain; Fr: fructose; GPx4: phospholipid hydroperoxide glutathione peroxidase 4; GR: glutathione reductase; GSH: reduced glutathione; GSSG: oxidized glutathione; HEPES: 4- (2-hydroxyethyl)-1-piperazineethanesulfonic acid; HF + Fr: high fat plus fructose; HF: high fat; MDA: malondialdehyde; MGO: methylglyoxal; MOPS: 3- (N-Morpholino)propanesulfonic acid; NAFLD: non-alcoholic fatty liver disease; NASH: non-alcoholic steatohepatitis; RCR: respiratory control ratio; ROS: reactive oxygen species; TBA: thiobarbituric acid; TBARS: thiobarbituric acid reactive substances; TC: total cholesterol; TG: triglycerides}

\section{Acknowledgements}

English language assistance was provided by Fidelmar Cortés Pérez.

\section{Funding}

This work was funded by a grant from Programa de Investigación 2018-2019 de la Coordinación de la Investigación Científica, Universidad Michoacana de San Nicolás de Hidalgo, Morelia, Michoacán, México (3757633 to CC-R).

\section{Availability of data and materials}

The datasets analyzed during the current study are available from the corresponding author on reasonable request.

\section{Authors' contributions}

CC-R and ARR-O conceived and designed the study. CIG-B, MAV-V and OO-A prepared and administered the diets, prepared the livers for histological analyses, performed the experiments in mitochondria and measured blood biochemical parameters. BAR-T and CG-L analyzed and interpreted the histological preparations. CC-R, AS-M and ARR-O analyzed and interpreted the data, CC-R and ARR-O drafted the manuscript. All authors read and approved the final manuscript.

\section{Ethics approval}

All the procedures with animals were performed according to the Federal Regulations for the Use and Care of Animals (NOM-062-ZOO-1999) issued by the Mexican Ministry of Agriculture. The experimental protocol was also approved by the Institutional Committee for Use of Animals of the Universidad Michoacana de San Nicolás de Hidalgo.

Consent for publication

Not applicable.

Competing interests

The authors declare that they have no competing interests. 


\section{Publisher's Note}

Springer Nature remains neutral with regard to jurisdictional claims in published maps and institutional affiliations.

\begin{abstract}
Author details
${ }^{1}$ Instituto de Investigaciones Químico-Biológicas, Universidad Michoacana de San Nicolás de Hidalgo, Edificio B-3, Ciudad Universitaria, 58030 Morelia, Michoacán, Mexico. ${ }^{2}$ Hospital General Regional 36, Instituto Mexicano del Seguro Social - IMSS, 72090 Puebla, Puebla, Mexico. ${ }^{3}$ Facultad de Ciencias Médicas y Biológicas "Dr. Ignacio Chávez", Universidad Michoacana de San Nicolás de Hidalgo, 58020 Morelia, Michoacán, Mexico. ${ }^{4}$ Hospital Regional de Alta Especialidad del Instituto de Seguridad y Servicios Sociales de los Trabajadores del Estado - ISSSTE, Carr. Morelia-Atapaneo Km 6, Atapaneo, 58300 Morelia, Michoacán, Mexico.
\end{abstract}

Received: 27 December 2018 Accepted: 21 March 2019

Published online: 30 March 2019

\section{References}

1. Chalasani N, Younossi Z, Lavine JE, Diehl AM, Brunt EM, Cusi K, Charlton M, Sanyal AJ. The diagnosis and management of non-alcoholic fatty liver disease: practice guideline by the American Gastroenterological Association, American Association for the Study of Liver Diseases, and American College of Gastroenterology. Gastroenterology. 2012;142:1592-609.

2. Loomba R, Sanyal AJ. The global NAFLD epidemic. Nat Rev Gastroenterol Hepatol. 2013;10:686-90.

3. Cohen JC, Horton JD, Hobbs HH. Human fatty liver disease: old questions and new insights. Science. 2011;332:1519-23.

4. Chanmugam P, Guthrie JF, Cecilio S, Morton JF, Basiotis PP, Anand R. Did fat intake in the United States really decline between 1989-1991 and 19941996? J Am Diet Assoc. 2003;103:867-72.

5. Said A, Ghufran A. Epidemic of non-alcoholic fatty liver disease and hepatocellular carcinoma. World J Clin Oncol. 2017;8:429-36.

6. Abdelmalek MF, Suzuki A, Guy C, Unalp-Arida A, Colvin R, Johnson RJ, Diehl AM. Increased fructose consumption is associated with fibrosis severity in patients with nonalcoholic fatty liver disease. Hepatology. 2010;51:1961-71.

7. Lanaspa MA, Sanchez-Lozada LG, Choi YJ, Cicerchi C, Kanbay M, RoncalJimenez CA, Ishimoto T, Li N, Marek G, Duranay M, Schreiner G, RodriguezIturbe B, Nakagawa T, Kang DH, Sautin YY, Johnson RJ. Uric acid induces hepatic steatosis by generation of mitochondrial oxidative stress: potential role in fructose-dependent and -independent fatty liver. J Biol Chem. 2012; 287:40732-44.

8. Satapati S, Sunny NE, Kucejova B, Fu X, He TT, Méndez-Lucas A, Shelton JM, Perales JC, Browning JD, Burgess SC. Elevated TCA cycle function in the pathology of diet-induced hepatic insulin resistance and fatty liver. J Lipid Res. 2012:53:1080-92.

9. Sil R, Chakraborti AS. Oxidative inactivation of liver mitochondria in high fructose diet-induced metabolic syndrome in rats: effect of glycyrrhizin treatment. Phytother Res. 2016;30:1503-12.

10. Mantena SK, Vaughn DP, Andringa KK, Eccleston HB, King AL, Abrams GA, Doeller JE, Kraus DW, Darley-Usmar VM, Bailey SM. High fat diet induces dysregulation of hepatic oxygen gradients and mitochondrial function in vivo. Biochem J. 2009;417:183-93.

11. Wang L, Liu X, Nie J, Zhang J, Kimball SR, Zhang H, Zhang WJ, Jefferson LS, Cheng Z, Ji Q, Shi Y. ALCAT1 controls mitochondrial etiology of fatty liver diseases, linking defective mitophagy to steatosis. Hepatology. 2015;61:486-96.

12. Ajith TA. Role of mitochondria and mitochondria-targeted agents in nonalcoholic fatty liver disease. Clin Exp Pharmacol Physiol. 2018;45:413-21.

13. Crescenzo R, Bianco F, Coppola P, Mazzoli A, Tussellino M, Carotenuto R, Liverini G, lossa S. Fructose supplementation worsens the deleterious effects of short-term high-fat feeding on hepatic steatosis and lipid metabolism in adult rats. Exp Physiol. 2014;99:1203-13.

14. Zelman K. The great fat debate: a closer look at the controversy-questioning the validity of age-old dietary guidance. J Am Diet Assoc. 2011;111:655-8.

15. Stanhope KL. Sugar consumption, metabolic disease and obesity: the state of the controversy. Crit Rev Clin Lab Sci. 2016;5:52-67.

16. Reeves PG, Nielsen FH, Fahey GC Jr. AIN-93 purified diets for laboratory rodents: final report of the American Institute of Nutrition ad hoc writing committee on the reformulation of the AIN-76A rodent diet. J Nutr. 1993; 123:1939-51.
17. Saavedra-Molina A, Devlin TM. Effect of extra-and intra-mitochondrial calcium on citrulline synthesis. Amino Acids. 1997;12:293-8.

18. Le TT, Ziemba A, Urasaki Y, Brotman S, Pizzorno G. Label-free evaluation of hepatic microvesicular steatosis with multimodal coherent anti-stokes Raman scattering microscopy. PLoS One. 2012;7:e51092.

19. Buege JA, Aust SD. Microsomal lipid peroxidation. Methods Enzymol. 1978;52:302-10

20. Seki S, Kitada T, Yamada T, Sakaguchi H, Nakatani K, Wakasa K. In situ detection of lipid peroxidation and oxidative DNA damage in non-alcoholic fatty liver diseases. J Hepatol. 2002;37:56-62.

21. Alwahsh SM, Xu M, Seyhan HA, Ahmad S, Mihm S, Ramadori G, Schultze FC. Diet high in fructose leads to an overexpression of lipocalin-2 in rat fatty liver. World J Gastroenterol. 2014;20:1807-21.

22. George J, Pera N, Phung N, Leclercq I, Yun Hou J, Farrell G. Lipid peroxidation, stellate cell activation and hepatic fibrogenesis in a rat model of chronic steatohepatitis. J Hepatol. 2003;39:756-64

23. Skulachev VP. Bioenergetic aspects of apoptosis, necrosis and mitoptosis. Apoptosis. 2006;11:473-85

24. Farrell GC, van Rooyen D, Gan L, Chitturi S. NASH is an inflammatory disorder: pathogenic, prognostic and therapeutic implications. Gut Liver. 2012;6:149-71.

25. Armutcu F, Kanter M, Gurel A, Unalacak M. Excessive dietary fructose is responsible for lipid peroxidation and steatosis in the rat liver tissues. Turkiye Klinikleri J Med Sci. 2007;27:164-9.

26. Cozza G, Rossetto M, Bosello-Travain V, Maiorino M, Roveri A, Toppo S, Zaccarin M, Zennaro L, Ursini F. Glutathione peroxidase 4-catalyzed reduction of lipid hydroperoxides in membranes: the polar head of membrane phospholipids binds the enzyme and addresses the fatty acid hydroperoxide group toward the redox center. Free Radic Biol Med. 2017; 112:1-11.

27. Allaman I, Bélanger M, Magistretti PJ. Methylglyoxal, the dark side of glycolysis. Front Neurosci. 2015;9:23.

28. Park YS, Koh YH, Takahashi M, Miyamoto Y, Suzuki K, Dohmae N, Takio K, Honke $\mathrm{K}$, Taniguchi N. Identification of the binding site of methylglyoxal on glutathione peroxidase: methylglyoxal inhibits glutathione peroxidase activity via binding to glutathione binding sites Arg 184 and 185. Free Radic Res. 2003;37:205-11.

29. Murphy MP. How mitochondria produce reactive oxygen species. Biochem J. 2009:417:1-13

30. Kushnareva Y, Murphy AN, Andreyev A. Complex I-mediated reactive oxygen species generation: modulation by cytochrome $c$ and $N A D(P)^{+}$ oxidation-reduction state. Biochem J. 2002:368:545-53.

31. Latipää PM, Kärki TT, Hiltunen JK, Hassinen IE. Regulation of palmitoylcarnitine oxidation in isolated rat liver mitochondria. Role of the redox state of $\mathrm{NAD}(\mathrm{H})$. Biochim Biophys Acta. 1986;875:293-300.

32. Saheki T, Kobayashi $K$, lijima M, Horiuchi M, Begum L, Jalil MA, Li MX, Lu YB, Ushikai M, Tabata A, Moriyama M, Hsiao KJ, Yang Y. Adult-onset type II citrullinemia and idiopathic neonatal hepatitis caused by citrin deficiency: involvement of the aspartate glutamate carrier for urea synthesis and maintenance of the urea cycle. Mol Genet Metab. 2004;81(Suppl 1):20-6.

33. Tandra S, Yeh MM, Brunt EM, Vuppalanchi R, Cummings OW, Ünalp-Arida A, Wilson LA, Chalasani N. Presence and significance of microvesicular steatosis in nonalcoholic fatty liver disease. J Hepatol. 2011:55(3):654-9.

34. Nolan CJ, Damm P, Prentki M. Type 2 diabetes across generations: from pathophysiology to prevention and management. Lancet. 2011;378:169-81.

35. Ishimoto T, Lanaspa MA, Rivard CJ, Roncal-Jimenez CA, Orlicky DJ, Cicerchi C, McMahan RH, Abdelmalek MF, Rosen HR, Jackman MR, MacLean PS, Diggle CP, Asipu A, Inaba S, Kosugi T, Sato W, Maruyama S, Sánchez-Lozada LG, Sautin YY, Hill JO, Bonthron DT, Johnson RJ. High-fat and high-sucrose (western) diet induces steatohepatitis that is dependent on fructokinase. Hepatology. 2013:58:1632-43.

36. Huang BW, Chiang MT, Yao HT, Chiang W. The effect of high-fat and highfructose diets on glucose tolerance and plasma lipid and leptin levels in rats. Diabetes Obes Metab. 2004;6:120-6.

37. D'Angelo G, Elmarakby AA, Pollock DM, Stepp DW. Fructose feeding increases insulin resistance but not blood pressure in Sprague-Dawley rats. Hypertension. 2005;46:806-11.

38. Tillman EJ, Morgan DA, Rahmouni K, Swoap SJ. Three months of highfructose feeding fails to induce excessive weight gain or leptin resistance in mice. PLoS One. 2014;9:e107206.

39. Bocarsly ME, Powell ES, Avena NM, Hoebel BG. High-fructose corn syrup causes characteristics of obesity in rats: increased body weight, body fat and triglyceride levels. Pharmacol Biochem Behav. 2010;97:101-6. 
40. Michaelis OE 4th, Ellwood KC, Judge JM, Schoene NW, Hansen CT. Effect of dietary sucrose on the SHR/N-corpulent rat: a new model for insulinindependent diabetes. Am J Clin Nutr. 1984;39(4):612-8.

41. Schemmel RA, Teague RJ, Bray GA. Obesity in Osborne-Mendel and S 5B/PI rats: effects of sucrose solutions, castration, and treatment with estradiol or insulin. Am J Phys. 1982;243(3):R347-53.

42. Elliott SS, Keim NL, Stern JS, Teff K, Havel PJ. Fructose, weight gain, and the insulin resistance syndrome. Am J Clin Nutr. 2002;76:911-22.

43. Blasiole DA, Davis RA, Attie AD. The physiological and molecular regulation of lipoprotein assembly and secretion. Mol BioSyst. 2007;3:608-19.

44. Choi SS, Diehl AM. Hepatic triglyceride synthesis and nonalcoholic fatty liver disease. Curr Opin Lipidol. 2008;19:295.

45. Crescenzo R, Bianco F, Falcone I, Coppola P, Liverini G, lossa S. Increased hepatic de novo lipogenesis and mitochondrial efficiency in a model of obesity induced by diets rich in fructose. Eur J Nutr. 2013;52:537-45.

46. Alwahsh SM, Dwyer BJ, Forbes S, Thiel DH, Lewis PJ, Ramadori G. Insulin production and resistance in different models of diet-induced obesity and metabolic syndrome. Int J Mol Sci. 2017;18:E285.

47. Vos MB, Lavine JE. Dietary fructose in nonalcoholic fatty liver disease. Hepatology. 2013;57:2525-31.

48. Tilg $\mathrm{H}$, Moschen AR. Evolution of inflammation in nonalcoholic fatty liver disease: the multiple parallel hits hypothesis. Hepatology. 2010;52:1836-46.

49. https://www.labdiet.com/cs/groups/lolweb/@labdiet/documents/web_ content/mdrf/mdi4/ edisp/ducm04_028021.pdf. Accessed 11 Mar 2019.

Ready to submit your research? Choose BMC and benefit from:

- fast, convenient online submission

- thorough peer review by experienced researchers in your field

- rapid publication on acceptance

- support for research data, including large and complex data types

- gold Open Access which fosters wider collaboration and increased citations

- maximum visibility for your research: over $100 \mathrm{M}$ website views per year

At $\mathrm{BMC}$, research is always in progress.

Learn more biomedcentral.com/submissions 